\title{
Acesso ao alimento artificial e enchimento do trato digestivo de juvenis do camarão marinho Litopenaeus vannamei (Boone) (Crustacea, Decapoda, Penaeidae) durante as fases clara e escura do período de 24 horas
}

\author{
Cibele S. Pontes ${ }^{1,2} \&$ Maria de F. Arruda ${ }^{1}$ \\ Laboratório de Estudo do Comportamento de Camarões, Departamento de Fisiologia, Universidade Federal do Rio Grande \\ do Norte. Caixa Postal 1511, Campus Universitário, Lagoa Nova II, 59072-970 Natal, Rio Grande do Norte. \\ E-mail: cspontes@natal.digi.com.br \\ ${ }^{2}$ Bolsista de Desenvolvimento Científico Regional, do CNPq.
}

\begin{abstract}
Artificial food access and digestive tract filling of juvenil marine shrimp Litopenaeus vannamei (Boone) (Crustacea, Decapoda, Penaeidae) during light and dark phases in 24-hour period. The few data on the marine shrimp Litopenaeus vannamei (Boone, 1931) behavior related to feed offer in trays can result inadequate feeding by the animal, feed wastage and unnecessary nutrient input, increasing feed costs and potential environmental pollution of that culture. In order to provide tools for improved feed management methods in shrimp farms, a behavioral study was conducted, using 64 Litopenaeus vannamei juveniles $(7,57 \pm 1,01 g)$, one animal per 33 $\mathrm{m}^{2}$. They were submitted to artificial photoperiods, half of them in reversed cycle, in order to register behavior during light and dark phases. The following variables were registered (continuous sampling) after feed exposition: a) latency to access the feeding tray, b) latency to start eating and c) digestive tract filling. The animals accessed the feeding trays and started consumption faster in the light phase hours. The digestive tract filling was higher half hour following feed offer, specially in the hours during the light phase.
\end{abstract}

KEY WORDS. Applied ethology, behavior, feeding, shrimp culture.

RESUMO. A escassez de dados acerca do comportamento alimentar do camarão marinho Litopenaeus vannamei (Boone, 1931) com relação ao alimento artificial ofertado em bandejas poderá induzir a uma alimentação inadequada, resultando no desperdício da ração, no aporte desnecessário de nutrientes, e conseqüente aumento dos custos com a alimentação e da poluição ambiental potencial desta atividade. Objetivando fornecer subsídios para a melhoria do manejo alimentar praticado nas fazendas, foram realizados estudos comportamentais utilizando 64 juvenis de Litopenaeus vannamei $(7,57 \pm 1,01 \mathrm{~g})$, mantidos em densidade populacional de $33 \mathrm{~m}^{2}$, submetidos a fotoperíodo artificial, em ciclo invertido, para observação durante as fases clara e escura. A ração foi ofertada em intervalos pré-estabelecidos, registrando-se posteriormente através de método focal contínuo: a) latência para chegada à bandeja, b) latência para consumo do alimento e c) Índice de Enchimento do Trato Digestivo. Os animais foram mais rápidos para chegar à bandeja e também para iniciar o consumo do alimento nos horários da fase clara. $\mathrm{O}$ enchimento do trato digestivo mostrou-se superior na meia hora subsequente à oferta do alimento, especialmente nos horários da fase clara.

PALAVRAS CHAVE. Alimentação, carcinicultura, comportamento, etologia aplicada.

No meio aquático, a eficiência de determinado alimento depende em grande parte de o mesmo ser rapidamente reconhecido e aceito, devido à rápida lixiviação de seus nutrientes (Costero \& Meyers 1993). Da mesma forma, devemos considerar que, no ambiente de cultivo, o consumo irá variar em função das respostas comportamentais da espécie cultivada frente ao alimento artificial ofertado, ao longo de $24 \mathrm{~h}$.

Com relação ao comportamento, RoBERTSON et al. (1993), afirmam que a maior parte dos camarões encontrados na natu- reza possui sulco adrostral e apresenta um padrão de atividade similar: são tipicamente ativos à noite e se entocam durante o dia. Em contraste, segundo Pérez-Farfante (1969), algumas espécies de camarão branco sem sulco adrostral, como é o caso de Litopenaeus vannamei (Boone, 1931), normalmente não se enterram durante o dia e são ativos de dia e de noite.

As pesquisas relacionadas ao comportamento alimentar de peneídeos abordam aspectos hierárquicos das respostas desses animais frente ao estímulo químico apresentado (Costero 
\& Meyers 1993, Pittet et al. 1996). Por outro lado, trabalhos referentes à alimentação de camarões são realizados em sua maioria em viveiros de cultivo e geralmente avaliam diferentes tipos de alimentos artificiais ou manejos alimentares, estimando os resultados com base em variáveis indicativas, como ganho de peso, crescimento e sobrevivência dos animais (SEDGWICK 1979, Robertson et al. 1993, Martinez-Cordova et al. 1998, Velasco et al. 1999, Nunes \& PARsons 1999, TACon et al. 2002, SMith et al. 2002, Cuzon et al. 2004).

No Brasil, a grande maioria dos criadores de camarão adota metodologia de cultivo semi-intensivo, caracterizada por densidades populacionais relativamente moderadas $(20$ a 30 camarões $/ \mathrm{m}^{2}$ ), pela utilização de aeradores em horários críticos de diminuição do oxigênio dissolvido na água e também pelo uso de ração como complemento ao alimento natural presente no viveiro. $\mathrm{O}$ consumo da ração adicionada aos viveiros é monitorado empiricamente através da utilização de bandejas. A quantidade de bandejas por hectare e horários de distribuição do alimento variam de acordo com critérios, relativos às condições de cultivo, definidos pelo cultivador, sendo inexistentes estudos comportamentais dos camarões com relação ao alimento artificial ofertado em bandejas.

No Nordeste do Brasil, as bandejas de alimentação são estruturas circulares fabricadas com a borda interna de pneus ("virola"), na base das quais é afixada uma tela de 1,3 $\mathrm{mm}$ de diâmetro. As bandejas são distribuídas por toda a extensão do viveiro, presas em varas fincadas, numa densidade de 30 unidades/hectare (NunEs \& SANDoval 1997), havendo casos onde a quantidade utilizada extrapola o dobro desse número em função da densidade populacional do cultivo.

Com o objetivo de fornecer subsídios para a melhoria do manejo alimentar praticado nas fazendas de cultivo, este trabalho avalia qual a fase do dia e horário em que o camarão marinho L. vannamei juvenil apresenta maior propensão para o acesso e ingestão da ração ofertada em bandeja. Neste sentido, o conhecimento da latência para chegada ao alimento artificial e para o consumo do alimento, bem como do Índice de Enchimento do Trato Digestivo, poderão ser utilizados como uma eficiente ferramenta.

\section{MATERIAL E MÉTODOS}

Foram utilizados 64 juvenis da espécie L. vannamei, com dois meses $(7,57 \pm 1,01 \mathrm{~g})$, provenientes de viveiro de cultivo, marcados com anéis de silicone de diferentes cores no pedúnculo ocular para reconhecimento individual. Os experimentos foram desenvolvidos em laboratório, sendo utilizados quatro aquários (50 x $30 \times 40 \mathrm{~cm}$ ) com $30 \mathrm{~L}$ de água do mar cada, em sistema fechado de recirculação de água, com aeração constante e filtração contínua através de cascalho e conchas de ostras quebradas.

Para possibilitar a observação simultânea dos comportamentos nas fases clara e escura, os aquários foram submetidos a ciclo artificial invertido através de interruptor horário, perma- necendo dois deles no claro e dois no escuro (7:00 às 19:00 h). Iluminados individualmente com lâmpadas de 15 watts fluorescente branca para a fase clara e incandescente vermelha (Rodriguez \& Naylor 1972, Hindley 1975) para a escura, os aquários permaneceram sob luminosidades médias de 57 lux e um lux, respectivamente.

Os animais foram colocados em número de quatro por aquário, havendo duas réplicas ao longo do tempo, totalizando oito aquários e 32 indivíduos observados para cada uma das fases (claro e escuro). As observações duraram 33 dias para cada réplica e foram iniciadas após nove dias de adaptação dos animais às condições experimentais. Os registros foram realizados por dois observadores, cada um contemplando aleatoriamente dois aquários por dia, um na fase clara e outro na escura. Como regra geral, os indivíduos eram observados durante 15 minutos ( $\mathrm{A}=$ antes da oferta do alimento), e, após um intervalo de 30 minutos, a ração era ofertada. A partir de então, eram observados nos 30 minutos imediatamente posteriores a oferta $(=1)$ e, após outro intervalo de 30 minutos, voltavam a ser observados por mais 30 minutos (=2), totalizando $480 \mathrm{~h}$ de observação em todo o experimento. Para efeito de análise, os horários referentes à fase clara foram considerados como manhã (M) e tarde (T), e os concernentes à escura como início da noite (IN) e final da noite (FN).

Os camarões foram alimentados com ração peletizada própria para seu cultivo (35\% de proteína bruta), apenas na fase em que eram observados, com o equivalente a $10 \%$ da biomassa total presente no aquário. A ração foi ofertada em bandejas de acrílico transparente $(4 \times 3 \times 2 \mathrm{~cm})$ duas vezes ao dia, uma e nove horas após o início da fase clara ou escura. Foram observadas, através de método focal contínuo, as variáveis: a) latência para chegada à bandeja, b) latência para consumo do alimento e c) Índice de Enchimento do Trato Digestivo (IETD).

Com relação à latência, as medições foram realizadas para cada indivíduo conforme definição dada por MARTIN \& BATESON (1993), como sendo o tempo decorrido entre algum evento específico, ao início da ocorrência do comportamento. Neste caso, registrou-se o tempo ocorrido desde a apresentação do estímulo (ração), até a chegada do animal à bandeja e até o início da ingestão do alimento. Uma vez que cada janela de observação durou meia hora, o tempo máximo de registro para latência foi de 1800 segundos.

Para avaliação do consumo do alimento, realizou-se inicialmente a padronização visual da quantidade de alimento presente na parte inicial do trato digestivo (proventrículo anterior), classificando-o com base nos seguintes estágios de enchimento: vazio $=0$, pouco alimento $=1$, enchimento médio $=2$, e cheio = 3 (Fig. 1A, B, C e D, respectivamente). Esse parâmetro foi registrado imediatamente antes da oferta do alimento e após 30 minutos de observação, calculando-se posteriormente o IETD, através da diferença entre a situação final e inicial (Fig. 1).

Uma vez constatada a não aderência dos dados à distribuição normal (Shapiro-Wilks) e a heterogeneidade nas

Revista Brasileira de Zoologia 22 (4): 1039-1043, dezembro 2005 


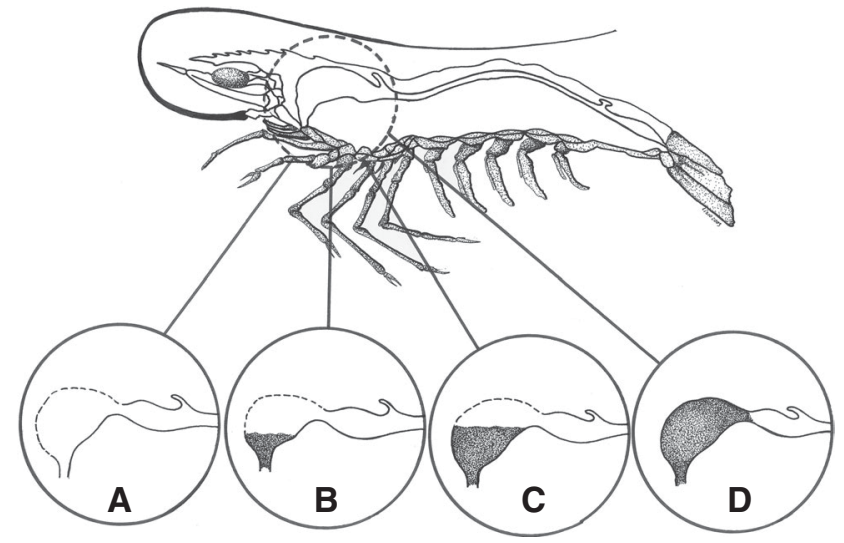

Figura 1. Padronização visual da situação do trato digestivo de $L$. vannamei através da observação do proventrículo anterior. (A) Vazio, (B) pouco, (C) médio, (D) cheio.

variâncias dos fatores (Levene), utilizou-se o teste de análise de variância não paramétrico de Kruskal-Wallis. Em caso de constatação de diferenças significativas aplicou-se o teste post hoc $\mathrm{U}$ de Mann-Whitney para comparação dois a dois (STEEL \& Torrie 1988). Adotou-se o nível de significância de 0,05 para avaliação dos resultados.

\section{RESULTADOS}

Devido à não normalidade de distribuição dos dados, os resultados serão graficamente representados através de sua mediana e amplitude interquartílica (75\% a 25\%).

\section{Latência para chegada à bandeja e consumo do alimento}

Verificou-se haver diferença na latência para chegada à bandeja de acordo com o horário de oferta da ração (KruskalWallis, $\mathrm{H}(93,15)=12,75 ; \mathrm{p}=0,00)$, que resultaram mais elevadas no início e final da noite (IN e FN), quando comparados com manhã e tarde (M e T) (Fig. 2). Da mesma forma, a latência para consumo do alimento (Kruskal-Wallis, H $(3,15)=32,10$; $\mathrm{p}=0,00)$ resultou semelhante nos horários da manhã e tarde $(\mathrm{M}$ e T), e por sua vez inferior ao início e final da noite (IN e FN) (Fig. 3).

\section{Índice de enchimento do trato digestivo}

O IETD mostrou-se superior na fase clara quando comparada à escura (Fig. 4) (Mann-Whitney, p =0,00), verificando-se também diferenças entre os horários de observação (KruskalWallis $\mathrm{H}(7,30)=632,9 ; \mathrm{p}=0,00)$. Nas primeiras meias horas posteriores à oferta da ração (1), seja na fase clara ou escura, os animais apresentaram IETD mais elevados que naquelas ocorridas uma hora após (2). Quando comparados os horários da fase clara com os da escura, foram observados valores semelhantes entre $1 \mathrm{M}$ e $1 \mathrm{~T}$, e entre $1 \mathrm{IN}$ e $1 \mathrm{FN}$, sendo os dois primeiros horários (fase clara) superiores aos dois últimos (escura) (Fig. 5).

\section{DISCUSSÃO}

Os resultados de latência apontam que $L$. vannamei chega à bandeja e inicia a ingestão da ração mais rapidamente na fase clara, pela manhã e à tarde, indicando que nestes horários os animais se encontram mais propícios à alimentação. Estas respostas podem ocorrer tanto devido a uma maior facilidade de localização do alimento em função da presença da luz, quanto a uma resposta fisiológica mais acentuada ao estímulo químico do alimento nesta fase.

De acordo com os resultados de IETD, os animais se alimentam tanto na fase clara quanto na escura e, embora o consumo da ração ocorra sobretudo na meia hora imediatamente posterior à sua oferta (em ambas as fases), o enchimento do trato é mais intenso na fase clara, pela manhã e à tarde. Uma hora após a oferta, a quantidade de alimento no proventrículo é mais reduzida.

Os trabalhos relacionados à utilização de bandejas para alimentação de camarões são contraditórios, e, embora apontem problemas associados a esta prática, são unânimes em ressaltar suas vantagens do ponto de vista ambiental e econômico. Segundo Nunes \& SANDOval (1997), apesar das bandejas servirem para a avaliação indireta do consumo, a concentração da ração em um único local pode induzir a desvantagens, como: atração de predadores (peixes e siris), aumento na competição por alimento, redução da área disponível para a alimentação natural dos camarões, diminuição do efeito fertilizante da ração não consumida sobre a produtividade natural do viveiro e, finalmente, aumento dos custos de distribuição do alimento. Além disso, é possível que a concentração do alimento faça com que o camarão gaste mais tempo à procura da fonte alimentar (NunEs \& PARSONS 1999). São necessárias pesquisas que utilizem uma abordagem comportamental dos aspectos ambientais e fisiológicos envolvidos na alimentação dos camarões em bandejas, como densidades populacionais elevadas, ciclo de muda, qualidade da água do cultivo, etc.

Nunes \& PARSONS (1999) verificaram que o conteúdo estomacal de Farfantepenaeus subtilis (Pérez-Farfante, 1967) foi mais elevado para animais alimentados com ração arremessada em comparação com alimentados em bandejas. Embora os animais não tenham apresentado diferença no crescimento e sobrevivência em função do método de alimentação, a distribuição uniforme (arremesso) foi responsável por uma maior facilidade de acesso e consumo do alimento, menor quantidade de estômagos vazios e maior proporção de alimento artificial no conteúdo estomacal.

Por outro lado, Martinez-CoRdova et al. (1998), relatam que L. vannamei alcançou maior ganho de peso com a alimentação em bandejas, em comparação com a distribuição por arremesso, que por sua vez foi responsável por maiores quantidades de matéria orgânica no solo do viveiro, maior demanda bioquímica de oxigênio e menores taxas de oxigênio dissolvido.

Corroborando nossos resultados relativos ao IETD, NUNES 

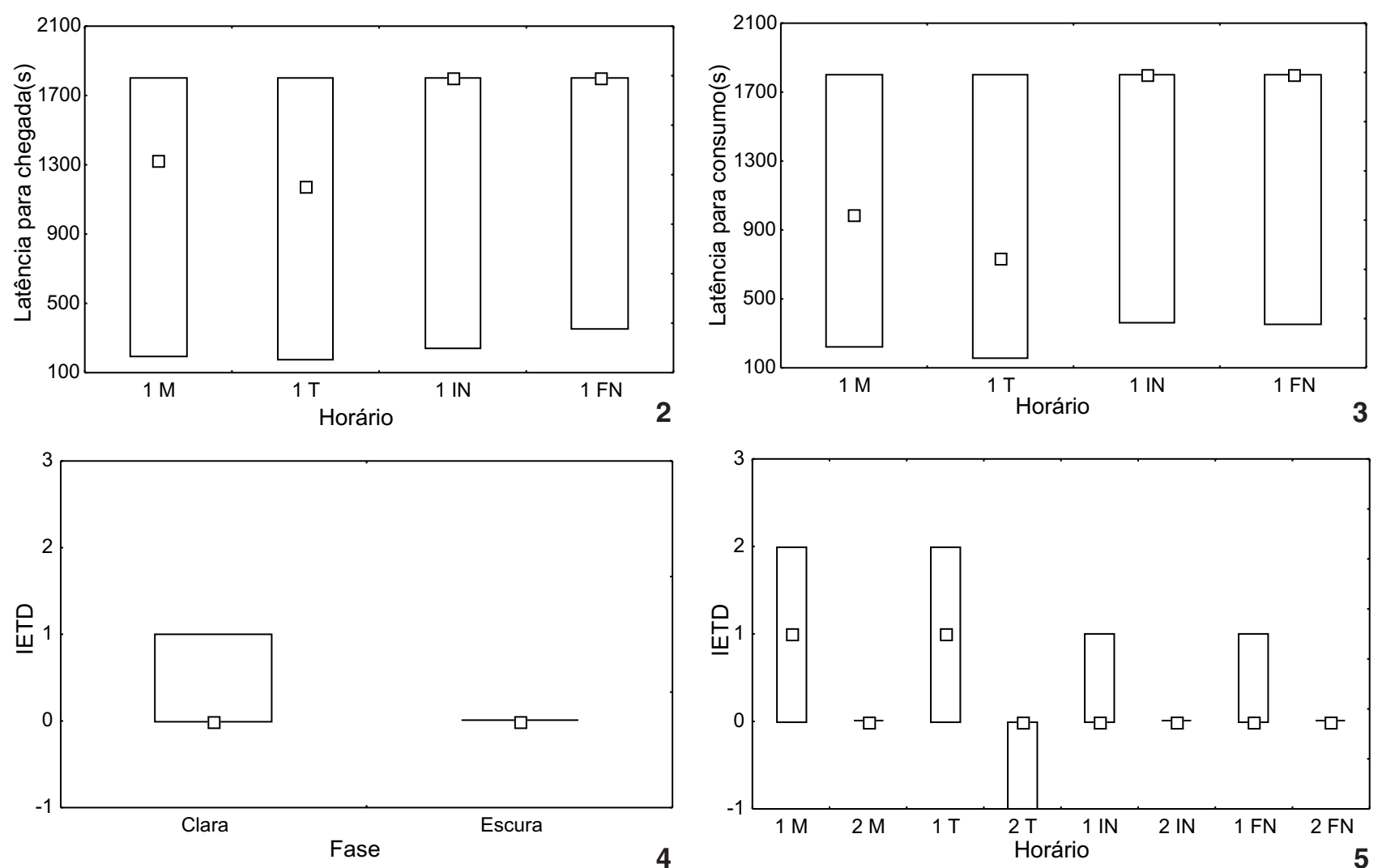

Figuras 2-5. Litopenaeus vannamei: (2-3) latência em segundos para a chegada à bandeja e para consumo do alimento de acordo com o horário de observação; (4-5) índice de enchimento do trato digestivo (IETD) com a fase e o horário de observação: (1) imediatamente após oferta, (2) uma hora após a oferta. (M) Manhã, (T) tarde, (IN) início da noite, (FN) final da noite.

et al. (1996) afirmam que juvenis de F. subtilis alimentam-se tanto durante o dia quanto à noite e que sempre ocorre o incremento da ingestão de alimento (artificial e natural) após a distribuição da ração no viveiro. Segundo estes autores, a variação na quantidade de alimento ingerido é afetada principalmente pela presença do alimento artificial, apesar de fatores externos (qualidade da água, intensidade luminosa, disponibilidade do alimento natural) e endógenos (ritmo alimentar natural da espécie), possam também influenciar.

Nunes et al. (1997) verificaram que o consumo alimentar é mais intenso nos 10 minutos posteriores à exposição à ração. Após esta fase, a ingestão passa a ocorrer de forma interrupta e pausada (captura do alimento, condução à boca, e consumo), podendo o camarão permanecer com o pelete ou parte dele em sua cavidade pré-oral, o que os autores relacionam à repleção do seu estômago. Adicionalmente, ocorre uma maior ocorrência de alimento no trato digestivo de $F$. subtilis trinta minutos após a sua distribuição (NunEs \& PARSONS 1999).

Neste estudo, constatamos que L. vannamei se alimenta sempre que há oferta de ração, embora haja variação na quantidade de ração ingerida em função da fase do dia (clara/escu- ra), sendo as menores latências para chegada e para consumo, bem como os maiores níveis de ingestão do alimento, ocorridos nos horários da fase clara. Considerando os resultados obtidos e as implicações ambientais e econômicas da alimentação noturna, recomendamos que o alimento artificial seja distribuído na fase clara do dia.

\section{AGRADECIMENTOS}

À CAPES, CNPq, Banco do Nordeste do Brasil, Cooperativa de Criadores Camarão do Rio grande do Norte, Fazenda de Carcinicultura Papeba e ao Departamento de Fisiologia da UFRN.

\section{REFERÊNCIAS BIBLIOGRÁFICAS}

Costero, M.T. \& S. Meyers. 1993. Evaluation of chemoreception by Penaeus vannamei under experimental condition. Progressive Fish-Culturist, Washington, 55 (3): 157-162.

Cuzon, G.; A. Lawrence; G. Gaxiola; C. Rosas \& J. Guillaume. 2004. Nutrition of Litopenaeus vannamei reared in tanks or in ponds. Aquaculture, Amsterdã, 235: 513-551. 
HindLEY, J.P.R. 1975. Effects of Endogenous and Some Exogenous Factors on the Activity of Juvenile banana Prawn Penaeus merguiensis. Marine Biology, Berlin, 29: 01-08.

Martin, P. \& Bateson, P. 1993. Measuring Behaviour - An Introductory Guide. Cambridge, University Press, 222p.

Martinez-Cordova, L.R.; A. Porchas-Cornejo; H. VillarrealColemnares; J.A. Calderon-Perez \& J.N. Naranjo-Paramo. 1998. Evaluation of Three Feeding Strategies on the Culture of White Shrimp Penaeus vannamei Boone 1931 in low water exchange ponds. Aquacultural Engineering, Amsterdam, 17: 21-28.

Nunes, A.J.P. \& G.J. Parsons. 1999. Feeding Levels of the Southern Brown Shrimp Penaeus subtilis in Response to Food Dispersal. Journal of the World Aquaculture Society, Baton Rouge, 30 (3): 331-348.

Nunes, A.J.P. \& P.F.C. Sandoval. 1997. Dados de Produção e Qualidade de Água de um Cultivo Comercial Semi-intensivo dos Camarões Penaeus subtilis e Penaeus vannamei com a Utilização de Bandejas de Alimentação. Boletim do Instituto de Pesca, São Paulo, 24, 221-231.

Nunes, A.J.P.; T.C.V. Gesteira \& S. Goddard. 1997. Food Ingestion and assimilation by the Southern Brown shrimp Penaeus subtilis under semi-intensive culture in NE Brazil. Aquaculture, Amsterdam, 149: 121-136.

Nunes, A.J.P.; S. Goddard \& T.C.V. Gesteira. 1996. Feeding activity patterns of the Southern brown shrimp Penaeus subtilis under semi-intensive culture in NE Brazil. Aquaculture, Amsterdam, 44: 371-386.

Pérez-Farfante, I. 1969. Western Atlantic Shrimp of the Genus Penaeus. Fishery Bulletin, 67: 461-591.

Pittet, A.O.; J.C. Ellis \& P.G. Lee. 1996. Methodology for the identification and quantitative measurement of chemical stimulants for penaeid shrimp. Aquaculture Nutrition, Oxford, 2: 175-182.

RoberTson, L.; A.L. LaWrence \& F.L. Castille. 1993. Effect of feeding frequency and feeding time on Growth of Penaeus vannamei (Boone). Aquaculture and Fisheries Management 24: 0106.

Rodriguez, G. \& E. NAylor. 1972. Behavioural rhythms in littoral prawns. Journal of Marine Biology Association, London, 52: 81-95.

SeDGWICK, R. W. 1979. Effect of ration size and feeding frequency on the growth and food conversion of juvenile Penaeus merguiensis De Man. Aquaculture, Amsterdam, 16: 279-298.

SMith, D.M.; M.A. Burford; S.J. TABRETT; S.J. IRvin \& L. WARD. 2002. The effect of feeding frequency on water quality and growth of the black tiger shrimp (Penaeus monodon). Aquaculture, Amsterdam, 207: 125-136.

Steel, R.G.D. \& J.H. Torrie. 1988. Bioestadística: Principios e Procedimientos. Mexico, McGraw-Hill de Mexico, $2^{\text {a }}$ ed., $622 \mathrm{p}$.

Tacon, A.J.G.; J.J. Cody; L.D. Conquest; S. Divakaran; I.P. Forster \& O.E. Decamp. 2002. Effect of culture system on the nutrition and growth performance of Pacific White shrimp Litopenaeus vannamei (Boone) fed different diets. Aquaculture Nutrition, Oxford, 8: 121-137.

Velasco, M.; A.L. Lawrence \& F.L. Castille. 1999. Effect of variations in daily feeding frequency and ration size on growth of shrimp Litopenaeus vannamei (Boone), in zerowater exchange culture tanks. Aquaculture, Amsterdam, 179: 141-148.

Recebido em 11.X.2005; aceito em 07.XI.2005. 